\title{
Unified dispersive approach to real and virtual photon-photon scattering into two pions
}

\section{B. Moussallam*}

Groupe de physique théorique, IPN bât. 100, Université Paris-Sud 11

E-mail: moussall@ipno.in2p3.fr

\begin{abstract}
A representation of the amplitude $\gamma^{*}\left(q^{2}\right) \rightarrow \gamma \pi \pi$ is proposed which combines large $N_{c}$ chiral resonance Lagrangian modelling with general unitarity and analyticity properties. The amplitude is constrained from $\gamma \gamma$ scattering results and $e^{+} e^{-} \rightarrow \gamma \pi^{0} \pi^{0}$ measurements by the CMD-2 and SND collaborations. As an application, the contributions of the $\pi \pi+\gamma$ states to the muon $g-2$ via the hadronic vacuum polarization are reconsidered, taking into account the effect of the strong $S$-wave $\pi \pi$ rescattering in a model independent way.
\end{abstract}

International Conference on the Structure and the Interactions of the Photon including the 20th International Workshop on Photon-Photon Collisions and the International Workshop on High Energy Photon Linear Colliders

20 - 24 May 2013

Paris, France

${ }^{*}$ Speaker. 


\section{Introduction}

There is a persistent discrepancy, at the three sigma level, for the muon anomalous magnetic moment $a_{\mu}=(g-2) / 2$ between the experimental value [1] and the theoretical one computed in the standard model (see e.g. [2] for a review). This calls for a careful re-examination of the parts of the calculation in which the strong interaction is involved. The leading contribution is associated with the hadronic vacuum polarization (HVP) function, and the contribution from $\pi^{+} \pi^{-}$, proportional to the square of the pion form factor, dominates the HVP unitarity relation. This has triggered experimental efforts for measuring the pion form factor to high accuracy, in particular, via the initial-state radiation (ISR) method (see $[3,4]$ and references therein). In the $e^{+} e^{-} \rightarrow$ $\gamma \pi^{+} \pi^{-}$cross-section, the final-state radiation (FSR) amplitude contributes in addition to the ISR. In principle, they could be separated experimentally by performing a partial-wave analysis. The FSR amplitude is also needed for computing the $\gamma \pi \pi$ contribution in the HVP unitarity relation. In practice, the FSR amplitude is often estimated using the scalar QED (SQED) approximation, which treats the final-state pions as point-like and non-interacting. This approximation ignores, in particular, the influence of the strong $\pi \pi S$-wave attraction at low energy. A modelling of this effect using a narrow $\sigma$-meson gives surprisingly large results [5]. We discuss here an approach in which $\pi \pi$ rescattering is treated in the model independent Omnès method [6]. It can be viewed as a generalization of classic work on the $\gamma \gamma \rightarrow \pi \pi$ amplitude $[7,8,9,10]$ and uses $\gamma \gamma$ scattering experimental results as constraints. A further generalization to the case of two virtual photons is being studied [11], which will be applied to the light-by-light hadronic contribution to the muon $g-2$.

\section{Analyticity of partial-waves when $q^{2} \neq 0$}

Unitarity alone leads to the well-known Fermi-Watson phase relation. This relation, however, has restricted applicability: it applies to $\gamma^{*}\left(q^{2}\right) \gamma \rightarrow \pi \pi$ when $q^{2}<4 m_{\pi}^{2}$ but not to $\gamma^{*}\left(q^{2}\right) \gamma \rightarrow \pi \pi$ with $q^{2}>4 m_{\pi}^{2}$ [12]. The Omnès method applies to partial-wave projected amplitudes, it combines the unitarity relation and analyticity properties. We restrict ourselves here to the elastic scattering region $s \lesssim 1 \mathrm{GeV}^{2}$ which will limit the applicability of the amplitude to virtualities $q^{2} \lesssim 1 \mathrm{GeV}^{2}$. In the case of two real photons, the partial-wave amplitude is an analytic function of the $\pi \pi$ energy $s$, except for two cuts, the right-hand cut which lies on $\left[4 m_{\pi}^{2}, \infty\right]$ and the left-hand cut on $[-\infty, 0]$. The discontinuity across the right-hand cut is given by the unitarity relations, these have exactly the same form for $\gamma \gamma$ and $\gamma^{*} \gamma$ amplitudes. In contrast, the left-hand cuts differ. The main issue is to properly define this cut and verify that no anomalous threshold is present.

The left-hand cut is associated with singularities of the unprojected amplitude in the crossed channels. One firstly has the pion pole in the $\gamma^{*} \pi^{+} \rightarrow \gamma \pi^{+}$amplitude (so-called Born amplitude) the $J=0$ projection reads,

$$
h_{0,++}^{\text {Born }}\left(s, q^{2}\right)=\frac{F_{\pi}^{v}\left(q^{2}\right)}{s-q^{2}}\left[\frac{4 m_{\pi}^{2}}{\sigma_{\pi}(s)} L_{\pi}(s)-2 q^{2}\right], L_{\pi}(s)=\log \frac{1+\sigma_{\pi}(s)}{1-\sigma_{\pi}(s)}
$$

with $\sigma_{\pi}(s)=\sqrt{1-4 m_{\pi}^{2} / s}$. Having $q^{2} \neq 0$ affects the amplitude through the pion form factor $F_{\pi}^{v}\left(q^{2}\right)$ but not only. The singularities are modified: the Born amplitude displays a pole at $s=q^{2}$ in 
addition to a left-hand cut. Using the $q^{2}+i \varepsilon$ prescription moves the pole away from the right-hand cut when $q^{2}>4 m_{\pi}^{2}$. Secondly, one must consider the cuts associated with $\gamma^{*} \pi \rightarrow n \pi \rightarrow \gamma \pi$. These processes are expected to display sharp resonance effects below $1 \mathrm{GeV}$ from the vector mesons $\rho, \omega$. We may start with a large $N_{c}$ approximation, where resonances generate simple poles in $\gamma^{*} \pi \rightarrow \gamma \pi$ (note that scalar mesons, which violate large $N_{c}$ rules are not allowed in this channel). Using a resonance chiral Lagrangian, the contributions from a vector meson exchange to the three independent invariant amplitudes read

$$
\begin{aligned}
& A^{V}\left(s, t, q^{2}\right)=\tilde{C}_{V} F_{V \pi}\left(q^{2}\right)\left[\frac{s-4 m_{\pi}^{2}-4 t+q^{2}}{t-M_{V}^{2}}+\frac{s-4 m_{\pi}^{2}-4 u+q^{2}}{u-M_{V}^{2}}\right] \\
& B^{V}\left(s, t, q^{2}\right)=\tilde{C}_{V} F_{V \pi}\left(q^{2}\right)\left[\frac{1}{2\left(t-M_{V}^{2}\right)}+\frac{1}{2\left(u-M_{V}^{2}\right)}\right] \\
& C^{V}\left(s, t, q^{2}\right)=\tilde{C}_{V} F_{V \pi}\left(q^{2}\right)\left[\frac{1}{t-M_{V}^{2}}-\frac{1}{u-M_{V}^{2}}\right]
\end{aligned}
$$

The main difference when $q^{2} \neq 0$ is from the kinematics: for $\gamma \gamma \rightarrow \pi \pi$ the Mandelstam variables $t, u$ are negative while for $\gamma^{*}\left(q^{2}\right) \rightarrow \gamma \pi \pi$ they lie in the range: $\left[4 m_{\pi}^{2},\left(\sqrt{q^{2}}-m_{\pi}\right)^{2}\right]$. One must then take the width of the resonance into account, and this must be done in a way consistent with the general analyticity properties for, otherwise, the Omnès method would not be applicable. This may be implemented by using a Källén-Lehmann representation, i.e. by replacing, in eq. (2.2)

$$
\frac{1}{M_{V}^{2}-w} \longrightarrow \widetilde{B W}_{V}(w)=\frac{1}{\pi} \int_{4 m_{\pi}^{2}}^{\infty} d t^{\prime} \frac{\sigma\left(t^{\prime}, M_{V}, \Gamma_{V}\right)}{t^{\prime}-w}, \quad w=t, u
$$

One can use for the spectral function $\sigma\left(s, M_{V}, \Gamma_{V}\right)$ e.g. the imaginary part of an ordinary BreitWigner function with an energy dependent width. The function $\widetilde{B W}_{V}$ has a cut instead of a pole on the first Riemann sheet, while a pole appears on the second sheet. The cut structure of the partial-wave projection of the vector-exchange amplitude is illustrated on figs. 1: the left figure shows that the cut extends into the complex plane and approaches the right-hand cut. The vicinity of the right-hand cut is illustrated on the right figure. Thanks to the analytic propagator and the $q^{2}+i \varepsilon$ prescription, no intersection actually occurs (it can be shown that the cut crosses the real axis at a point strictly below $4 m_{\pi}^{2}$ ), which guarantees the absence of an anomalous threshold and the applicability of the usual Omnès method.

\section{The master formula for rescattering}

In order to discuss rescattering one considers amplitudes which correspond to definite $\pi \pi$ isospin, $\gamma^{*} \rightarrow \gamma(\pi \pi)_{I}$, with $I=0,2$. They are related to the $\pi^{+} \pi^{-}$and $\pi^{0} \pi^{0}$ amplitudes by

$$
\left(\begin{array}{c}
\sqrt{2} H_{\lambda \lambda^{\prime}}^{c} \\
H_{\lambda \lambda^{\prime}}^{n}
\end{array}\right)=\left(\begin{array}{cc}
-\sqrt{\frac{2}{3}} & -\sqrt{\frac{1}{3}} \\
-\sqrt{\frac{1}{3}} & \sqrt{\frac{2}{3}}
\end{array}\right)=\left(\begin{array}{c}
H_{\lambda \lambda^{\prime}}^{0} \\
H_{\lambda \lambda^{\prime}}^{2}
\end{array}\right) .
$$

We consider an Omnès representation for the isospin amplitudes based on twice-subtracted dispersion relations i.e. involving two polynomial parameters for each isospin $I$. These parameters, which depend on $q^{2}$, account for the effects of higher mass resonances (like the axial-vector or 

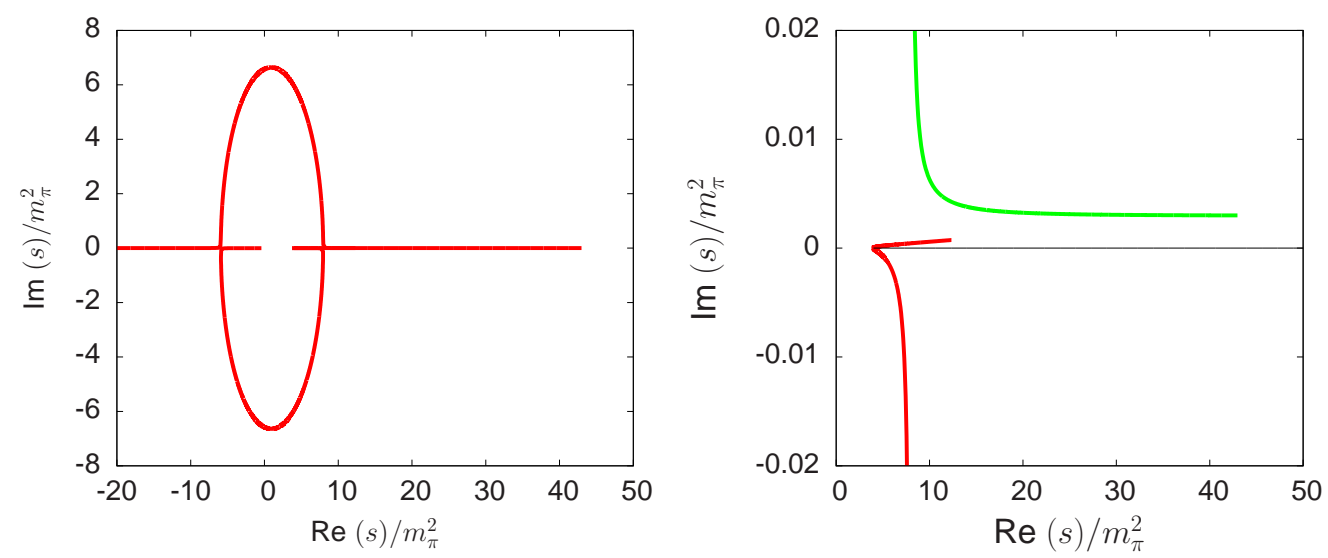

Figure 1: Cut structure of a partial-wave projection of the vector-exchange amplitude.

the tensor mesons) not explicitly included as well as the effects of inelasticity in the higher energy portions of integrals. Beside the properties of analyticity and elastic unitarity, there are additional physical constraints that must be imposed. Gauge invariance imposes that the amplitudes minus the Born term must vanish in the soft photon limit [13] which eliminates one of the parameters for each $I$. The helicity amplitude $H_{++}^{I}\left(s, q^{2}, z\right)$, where $z$ is the cosine of the scattering angle in the $\pi \pi$ CMS, can be written as

$$
H_{++}^{I}\left(s, q^{2}, z\right)=H_{++}^{I, \text { Born }}\left(s, q^{2}, z\right)+\sum_{V=\rho, \omega} H_{++}^{I, V}\left(s, q^{2}, z\right)+H_{++}^{I, \text { resc }}\left(s, q^{2}, z\right)
$$

The last term in eq. (3.2) accounts for the rescattering in the $J=0$ partial-wave, it reads

$$
\begin{gathered}
H_{++}^{I, \text { resc }}\left(s, q^{2}, z\right)=\Omega_{0}^{I}(s)\left\{\left(s-q^{2}\right) b^{I}\left(q^{2}\right)+s F_{\pi}^{v}\left(q^{2}\right)\left[\frac{s\left(J^{I, \pi}\left(s, q^{2}\right)-J^{I, \pi}\left(q^{2}, q^{2}\right)\right)}{s-q^{2}}\right.\right. \\
\left.\left.-q^{2} \hat{\boldsymbol{J}}^{I, \pi}\left(q^{2}\right)\right]+s \sum_{V=\rho, \omega} F_{V \pi}\left(q^{2}\right)\left[s J^{I, V}\left(s, q^{2}\right)-q^{2} J^{I, V}\left(q^{2}, q^{2}\right)\right]\right\},
\end{gathered}
$$

where $\Omega_{0}^{I}$ is the usual Omnès function, given in terms of the $\pi \pi$ scattering phase-shift $\delta_{0}^{I}$

$$
\Omega_{0}^{I}(s)=\exp \left[\frac{s}{\pi} \int_{4 m_{\pi}^{2}}^{\infty} d s^{\prime} \frac{\delta_{0}^{I}\left(s^{\prime}\right)}{s^{\prime}\left(s^{\prime}-s\right)}\right]
$$

and $J^{I, \pi}, J^{I, V}$ are the related integrals (see [6]) involving the partial-wave projections of the Born and the vector-exchange amplitudes respectively (without the form factor term),

$$
\begin{aligned}
& J^{I, \pi}\left(s, q^{2}\right)=\frac{1}{\pi} \int_{4 m_{\pi}^{2}}^{\infty} \frac{d s^{\prime}}{\left(s^{\prime}\right)^{2}\left(s^{\prime}-s\right)} \frac{\sin \delta_{0}^{I}\left(s^{\prime}\right)}{\left|\Omega_{0}^{I}\left(s^{\prime}\right)\right|} \bar{h}_{0,++}^{I, \pi}\left(s^{\prime}, q^{2}\right) \\
& J^{I, V}\left(s, q^{2}\right)=\frac{1}{\pi} \int_{4 m_{\pi}^{2}}^{\infty} \frac{d s^{\prime}}{\left(s^{\prime}\right)^{2}\left(s^{\prime}-s\right)} \frac{\sin \delta_{0}^{I}\left(s^{\prime}\right)}{\left|\Omega_{0}^{I}\left(s^{\prime}\right)\right|} \tilde{h}_{0,++}^{I, V}\left(s^{\prime}, q^{2}, m_{V}, \Gamma_{V}\right),
\end{aligned}
$$

Finally, $\hat{J}^{I, \pi}=\partial J^{I, \pi}\left(s, q^{2}\right) / \partial s$ at $s=q^{2}$. One notes that the integrations in eq. (3.5) are well defined since, as we have argued, the singularities of the partial-wave amplitudes do not overlap with the integration axis. The other two helicity amplitudes $H_{+-}, H_{+0}$ are affected by rescattering from $J \geq 2$ partial-waves: we have neglected this effect here since the $J=2$ phase-shifts $\delta_{2}^{I}$ are rather small for energies below $1 \mathrm{GeV}$. 


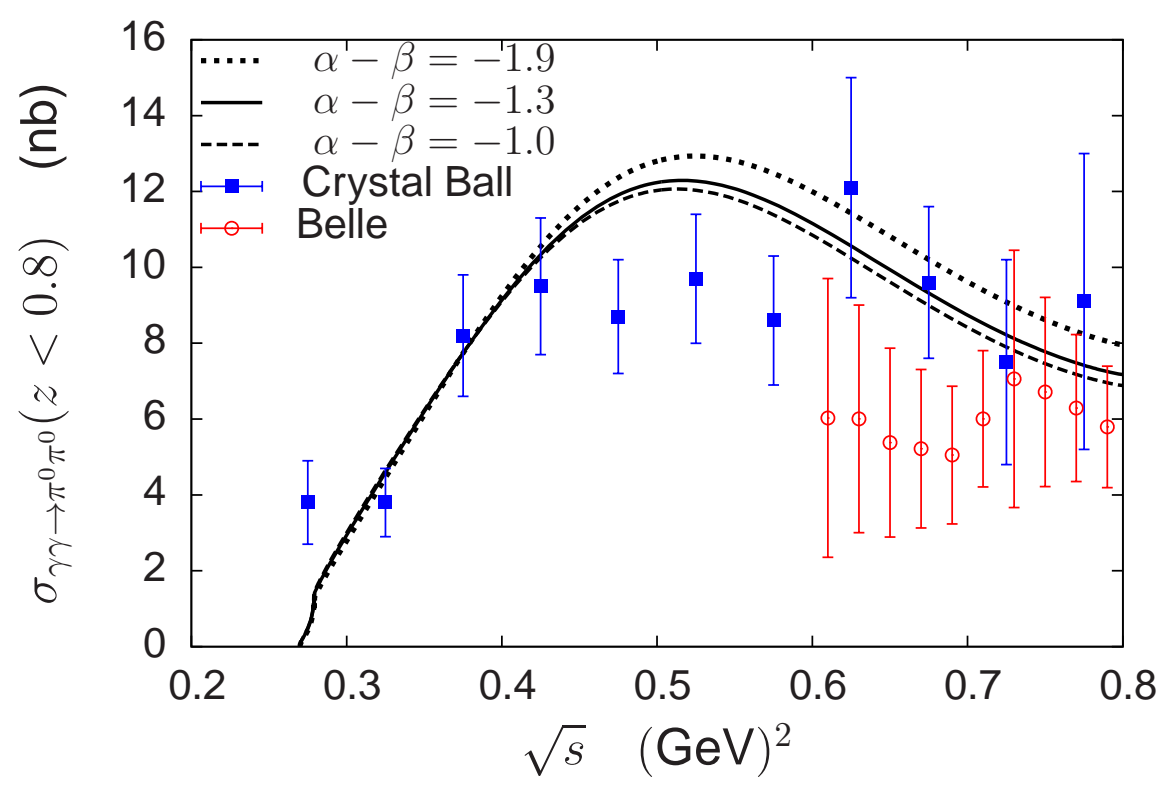

Figure 2: Comparison of the $\gamma \gamma \rightarrow \pi^{0} \pi^{0}$ cross-sections using the amplitude $H_{++}^{n}$ with $J=0$ rescattering and $H_{+-}^{n}=H_{+-}^{n, V}$, with experiment.

\section{Chiral and experimental constraints}

The two arbitrary functions $b^{0}\left(q^{2}\right), b^{2}\left(q^{2}\right)$, are constrained by chiral symmetry. In the exact chiral limit, the $\gamma^{*} \gamma$ amplitudes for producing a $\pi^{0}$ pair satisfies a soft pion theorem: the combination of the three invariant amplitudes $A(s, t)+2 q^{2}(B(s, t)-C(s, t))$ with $t=0$ vanishes at $s=0$ for any value of $q^{2}$. In the real world, this corresponds to an Adler zero at $s=s_{A}=O\left(m_{\pi}^{2}\right)$, depending on $q^{2}$, for the helicity amplitude $H_{++}^{n}$ with $t=m_{\pi}^{2}$. The correct chiral behaviour is enforced by matching the dispersive amplitudes for both $\pi^{0} \pi^{0}$ and $\pi^{+} \pi^{-}$with the corresponding chiral expansion expressions which are known at NLO $[14,15]$ at $s=0$ and small values of $q^{2}$. For larger values, in the range $q^{2} \lesssim 1 \mathrm{GeV}^{2}$, the dependence should be dominated by the light vector resonances. Introducing the combinations $b^{n}=\left(-b^{0}+\sqrt{2} b^{2}\right) / \sqrt{3}$ and $b^{c}=-\left(\sqrt{2} b^{0}+b^{2}\right) / \sqrt{6}$ corresponding to the $\pi^{0} \pi^{0}$ and $\pi^{+} \pi^{-}$channels, the following parametrization encodes these properties

$$
\begin{aligned}
& b^{n}\left(q^{2}\right)=b^{n}(0) F_{\chi}\left(q^{2}\right)+F_{R}\left(q^{2}\right), \\
& b^{c}\left(q^{2}\right)=b^{c}(0)+F_{R}\left(q^{2}\right)
\end{aligned}
$$

where

$$
F_{R}\left(q^{2}\right)=\beta_{\rho}\left(G S_{\rho}\left(q^{2}\right)-1\right)+\beta_{\omega}\left(B W_{\omega}\left(q^{2}\right)-1\right)
$$

involves the usual Gounaris-Sakurai and Breit-Wigner functions, and

$$
F_{\chi}\left(q^{2}\right)=12 m_{\pi}^{2}\left[\frac{m_{\pi}^{2}}{q^{2}} L_{\pi}^{2}\left(q^{2}\right)+\sigma_{\pi}\left(q^{2}\right) L_{\pi}\left(q^{2}\right)+3\right] .
$$

is generated from the ChPT matching. In the parametrization (4.1) we used the same resonance function for $b^{n}$ and $b^{c}$ i.e. we neglected the resonance contribution to $b^{2}$. This is justified from the 

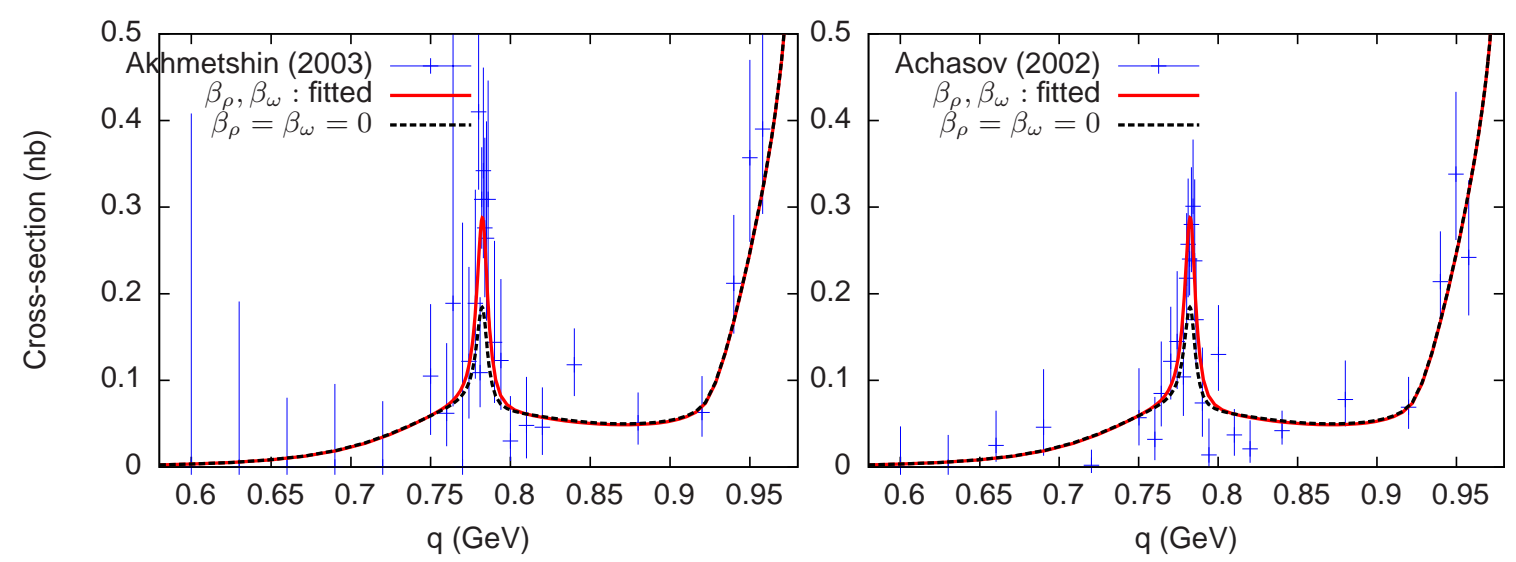

Figure 3: Experimental results for $\sigma\left(e^{+} e^{-} \rightarrow \gamma \pi^{0} \pi^{0}\right)$ and our two parameter fit.

fact that the Omnès functions satisfy the inequality $\left|\Omega_{2}(s)\right|<<\left|\Omega_{0}(s)\right|$ in the physically relevant region $4 m_{\pi}^{2} \leq s \leq q^{2}$ which suppresses the influence of $b^{2}$. Thanks to this simplification, determining the two parameters $\beta_{\rho}, \beta_{\omega}$ from $\sigma\left(e^{+} e^{-} \rightarrow \gamma \pi^{0} \pi^{0}\right)$ allows one to predict $\sigma^{F S R}\left(e^{+} e^{-} \rightarrow \gamma \pi^{+} \pi^{-}\right)$.

The values of $b^{n}(0), b^{c}(0)$ can be related to the polarizabilities of the $\pi^{+}$and the $\pi^{0}$ which parametrize the pion Compton amplitudes at threshold,

$$
\begin{aligned}
& \left(\alpha_{\pi^{0}}-\beta_{\pi^{0}}\right)=\frac{2 \alpha}{m_{\pi}}\left(b^{n}(0)-4 m_{\pi}^{2} \tilde{C}_{\rho^{0}} \widetilde{B W}_{\rho}\left(m_{\pi}^{2}\right)-4 m_{\pi}^{2} \tilde{C}_{\omega} B W_{\omega}\left(m_{\pi}^{2}\right)\right) \\
& \left(\alpha_{\pi^{+}}-\beta_{\pi^{+}}\right)=\frac{2 \alpha}{m_{\pi}}\left(b^{c}(0)-4 m_{\pi}^{2} \tilde{C}_{\rho^{+}} \widetilde{B W}_{\rho}\left(m_{\pi}^{2}\right)\right) .
\end{aligned}
$$

The values of the pion polarizabilities are not yet precisely determined experimentally, the status of the measurement under way at COMPASS is described in [16]. We will use here values compatible with the NNLO chiral calculations $[17,18]$. Fig. 2 shows the resulting prediction from our amplitude with $q^{2}=0$ for the $\gamma \gamma \rightarrow \pi^{0} \pi^{0}$ cross-section, compared to the experimental results from refs. [19, 20].

In order to address the case with $q^{2} \neq 0$ we have yet to specify the $q^{2}$ dependence of the three form factors which enter into the expression of the amplitude (3.2) (3.3). The pion form factor, of course, is known rather precisely from experiment. Some experimental data exist also for the $\omega \pi$ form factor in two kinematical regions surrounding the peak of the $\rho$ meson. The $F_{\rho \pi}$ form factor, finally, is more difficult to isolate experimentally than $F_{\omega \pi}$, because of the width of the $\rho$. We used the same type of modelling based on superposition of Breit-Wigner type functions together with symmetry arguments to fix the parameters. The experimental results for the $e^{+} e^{-} \rightarrow \gamma \pi^{0} \pi^{0}$ crosssections from the SND and CMD-2 collaborations [21, 22] can be reproduced rather well with our two-parameter Omnès amplitude. A combined fit to these two data sets gives for the parameters:

$$
\begin{aligned}
& \beta_{\rho}=0.05 \pm 0.09 \mathrm{GeV}^{-2} \\
& \beta_{\omega}=(-0.37 \pm 0.09) \cdot 10^{-1} \mathrm{GeV}^{-2}
\end{aligned}
$$

with $\chi^{2} / N_{d o f}=38 / 50$. Fig.3. shows the experimental and the fitted theoretical cross sections. 


\begin{tabular}{ll|c}
\hline channel & cross-section & $(g-2) / 2$ \\
\hline$\gamma \pi^{+} \pi^{-}$ & $\left|H^{\text {Born }}\right|^{2}$ & $41.9 \times 10^{-11}$ \\
$\gamma \pi^{+} \pi^{-}$ & $\left(H^{\text {Born }}\right)^{*} H^{V+r e s c}$ & $(1.31 \pm 0.30) \times 10^{-11}$ \\
$\gamma \pi^{+} \pi^{-}$ & $\left|H^{V+r e s c}\right|^{2}$ & $(0.16 \pm 0.05) \times 10^{-11}$ \\
$\gamma \pi^{0} \pi^{0}$ & $\left|H^{V+r e s c}\right|^{2}$ & $(0.33 \pm 0.05) \times 10^{-11}$ \\
\hline
\end{tabular}

Table 1: Central values of the $\pi \pi \gamma$ contributions (see eqs. (5.2) and (5.4) in the text) to the muon $g-2$ from the integration region $\sqrt{q^{2}} \leq 0.95 \mathrm{GeV}$.

\section{Application to the $\pi \pi \gamma$ contributions to the muon $g-2$}

The contribution of the hadronic vacuum polarization function to the muon $g-2$ has been expressed a long time ago by using the analyticity property of the polarization function $[23,24]$. It can be written in terms of physical $e^{+} e^{-} \rightarrow$ hadrons cross sections

$$
\left.\frac{g-2}{2}\right|_{H V P}=\frac{1}{4 \pi^{3}} \int_{4 m_{\pi}^{2}}^{\infty} d q^{2} K_{\mu}\left(q^{2}\right) \sum_{\text {hadrons }} \sigma_{e^{+} e^{-} \rightarrow \text { hadrons }}\left(q^{2}\right)
$$

(see e.g. [2] for the explicit expression of the function $K_{\mu}\left(q^{2}\right)$ ). A class of $O(\alpha)$ radiative corrections to the HVP contribution is generated by simply adding in eq. (5.1) the cross sections for $e^{+} e^{-} \rightarrow$ hadrons $+\gamma$, where the photon is emitted in the final state. The lightest hadrons $+\gamma$ state is $\pi^{0} \gamma$ which makes a contribution $(g-2) /\left.2\right|_{\pi^{0} \gamma}=(44.2 \pm 1.9) \cdot 10^{-11}$ (see [25]) of a size comparable to the error in the measurement of $a_{\mu}$. The next to lightest states are $\pi \pi \gamma$. When charged pions are present, the cross-section $\sigma_{e^{+} e^{-} \rightarrow \text { hadrons }+\gamma}^{F R}$ infrared divergent: in the case of $\pi^{+} \pi^{-} \gamma$ the divergence is easily seen to originate from the $1 /\left(q^{2}-s\right)^{2}$ denominator in $\left|H_{\lambda \lambda^{\prime}}^{\text {Born }}\right|^{2}$. As is well known, this divergence is cancelled by part of the $O(\alpha)$ radiative corrections to the $e^{+} e^{-} \rightarrow \pi^{+} \pi^{-}$ cross-section. Collecting these pieces together, one can then re-write the $\pi \pi \gamma$ contribution to the muon $g-2$ as a sum of three infrared finite quantities

$$
\left.\frac{g-2}{2}\right|_{\pi \pi \gamma}=\frac{1}{4 \pi^{3}} \int_{4 m_{\pi}^{2}}^{\infty} d q^{2} K_{\mu}\left(q^{2}\right)\left(\sigma_{e^{+} e^{-} \rightarrow \pi^{+} \pi^{-} \gamma}^{s Q E D}\left(q^{2}\right)+\hat{\sigma}_{e^{+} e^{-} \rightarrow \pi^{+} \pi^{-} \gamma}\left(q^{2}\right)+\sigma_{e^{+} e^{-} \rightarrow \pi^{0} \pi^{0} \gamma}\left(q^{2}\right)\right)
$$

where

$$
\sigma_{e^{+} e^{-} \rightarrow \pi^{+} \pi^{-} \gamma}^{s Q E D}=\frac{\alpha^{3}}{3 q^{2}} \sigma_{\pi}^{3}\left(q^{2}\right)\left|F_{\pi}^{v}\left(q^{2}\right)\right|^{2} \times \eta\left(q^{2}\right)
$$

(see e.g. [2] for the explicit expression of the function $\eta$ ) and $\hat{\sigma}$ is defined by simply removing the contribution proportional to the Born amplitude squared from the charged pions cross-section ( $\hat{\sigma}$ is thus not necessarily positive)

$$
\hat{\sigma}_{e^{+} e^{-} \rightarrow \pi^{+} \pi^{-} \gamma}=\frac{\alpha^{3}}{12\left(q^{2}\right)^{3}} \int_{4 m_{\pi}^{2}}^{q^{2}} d s\left(q^{2}-s\right) \sigma_{\pi}(s) \int_{-1}^{1} d z\left(S_{++}^{c}+S_{+-}^{c}+S_{+0}^{c}\right)
$$

where

$$
S_{\lambda \lambda^{\prime}}^{c}=2 \operatorname{Re}\left[\left(H_{\lambda \lambda^{\prime}}^{\text {Born }}\right)^{*}\left(H_{\lambda \lambda^{\prime}}^{c, V}+H_{\lambda \lambda^{\prime}}^{c, \text { resc }}\right)\right]+\left|H_{\lambda \lambda^{\prime}}^{c, V}+H_{\lambda \lambda^{\prime}}^{c, \text { resc }}\right|^{2} .
$$

The contributions to the muon $g-2$, restricting the integration range in eq. (5.2) to $\sqrt{q^{2}} \leq 0.95$ $\mathrm{GeV}$, within the domain of validity of the model, are shown in table 5. As compared to previous 
work, we find for the contribution linear in $H^{\text {Born }}$ (second line in the table) is positive, unlike the result of ref. [26]. This difference is partly due to the effect of the $\pi \pi$ rescattering, which was not taken into account in ref. [26] and partly to the somewhat larger integration range used in that work. The sum of the last two lines in the table can be compared with the calculation of ref. [5] based on an estimate of the $e^{+} e^{-} \rightarrow \gamma \sigma$ cross-section. We find that the $\sigma$-meson approximation to $\pi \pi$ rescattering in the present context, leads to an overestimate by a factor of three approximately.

\section{Conclusions}

We have proposed a theoretical model of the amplitude for producing a pion pair from one real plus one virtual photon. It combines the resonance chiral Lagrangian approach, which is used to describe resonance effects in the crossed channels $\gamma^{*} \pi \rightarrow \gamma \pi$ and the Omnès approach based on unitarity/analyticity for treating the $\pi \pi$ final-state interaction effects in the elastic regime. Our expression is a generalization of former work on photon-photon scattering $[7,8,9,10]$ and it reproduces the $\gamma \gamma \rightarrow \pi \pi$ amplitudes in the limit where the virtuality $q^{2}$ vanishes. We have shown that the usual Omnès formalism can be applied even in the region $q^{2}>4 m_{\pi}^{2}$ where the left and right-hand cuts seem to overlap, provided appropriate analytic forms of the resonance propagators in the crossed channels are used.

As an application, we have reconsidered the contribution from the $\gamma \pi \pi$ states in the hadronic vacuum polarization to the muon $g-2$ in the region $\sqrt{q^{2}}<0.95 \mathrm{GeV}$ and find some differences with several former approaches. However, in this region, the contribution is dominated by $\gamma \pi^{+} \pi^{-}$ and very well approximated by the sQED formula. Still, it would be of interest to be able to extend the integration range somewhat since one expects a kinematical increase of the FSR cross-section when $\sqrt{q^{2}}>m_{V}+m_{\pi}$ (with $V=\rho, \omega$, see fig. 3). This would necessitate to account for rescattering in $D$-waves as well as properly account for $\pi \pi-K \bar{K}$ inelasticity in the final state $S$-wave interaction.

Acknowledgements: Work supported in part by the European Community-Research Infrastructure Integrating Activity "Study of Strongly Integrating Matter" (acronym HadronPhysics3)

\section{References}

[1] D. Hanneke, S. Fogwell, and G. Gabrielse, New Measurement of the Electron Magnetic Moment and the Fine Structure Constant, Phys.Rev.Lett. 100 (2008) 120801, [arXiv: 0801 . 1134].

[2] F. Jegerlehner and A. Nyffeler, The Muon g-2, Phys.Rept. 477 (2009) 1-110, [arXiv:0 902 . 3360].

[3] KLOE Collaboration Collaboration, D. Babusci et al., Precision measurement of $\sigma\left(e^{+} e^{-} \rightarrow \pi^{+} \pi^{-} \gamma\right) / \sigma\left(e^{+} e^{-} \rightarrow \mu^{+} \mu^{-} \gamma\right)$ and determination of the $\pi^{+} \pi^{-}$contribution to the muon anomaly with the KLOE detector, Phys.Lett. B720 (2013) 336-343, [arXiv:1212 . 4524].

[4] BaBar Collaboration, J. Lees et al., Precise Measurement of the $e^{+} e^{-} \rightarrow \pi^{+} \pi^{-}(\gamma)$ Cross Section with the Initial-State Radiation Method at BABAR, Phys.Rev. D86 (2012) 032013, [arXiv: 1205 . 2228].

[5] S. Narison, Scalar mesons and the muon anomaly, Phys.Lett. B568 (2003) 231-236, [hep-ph/0303004].

[6] R. Omnès, On the Solution of certain singular integral equations of quantum field theory, Nuovo Cim. 8 (1958) 316-326. 
[7] M. Gourdin and A. Martin, Pion photon scattering, Nuovo Cim. 17 (1960) 224-243.

[8] R. Goble and J. L. Rosner, Soft-pion production in electron-positron collisions, Phys.Rev. D5 (1972) 2345-2358.

[9] D. Morgan and M. Pennington, Low-energy $\gamma \gamma \rightarrow \pi \pi$ Cross-section and the QED Born Amplitude, Phys.Lett. B192 (1987) 207.

[10] J. F. Donoghue and B. R. Holstein, Photon-photon scattering, pion polarizability and chiral symmetry, Phys.Rev. D48 (1993) 137-146, [hep-ph/9302203].

[11] M. Hoferichter, G. Colangelo, M. Procura, and P. Stoffer, Virtual photon-photon scattering, arXiv:1309.6877.

[12] M. J. Creutz and M. B. Einhorn, Pion pion scattering information from $e^{-} e^{+} \rightarrow \pi^{-} \pi^{+} \gamma$, Phys.Rev. D1 (1970) 2537.

[13] F. Low, Bremsstrahlung of very low-energy quanta in elementary particle collisions, Phys.Rev. 110 (1958) 974-977.

[14] J. F. Donoghue, B. R. Holstein, and Y. Lin, The Reaction $\gamma \gamma \rightarrow \pi^{0} \pi^{0}$ and Chiral Loops, Phys.Rev. D37 (1988) 2423.

[15] C. Unkmeir, S. Scherer, A. L'vov, and D. Drechsel, Generalized polarizabilities of the pion in chiral perturbation theory, Phys.Rev. D61 (2000) 034002, [hep-ph/9904442].

[16] COMPASS Collaboration, J. Friedrich, Chiral Dynamics and the Pion Polarisability: Measurements at COMPASS, PoS Bormio2013 (2013) 030.

[17] J. Gasser, M. A. Ivanov, and M. E. Sainio, Low-energy photon-photon collisions to two loops revisited, Nucl.Phys. B728 (2005) 31-54, [hep-ph/ 0506265$].$

[18] J. Gasser, M. A. Ivanov, and M. E. Sainio, Revisiting $\gamma \gamma \rightarrow \pi^{+} \pi^{-}$at low energies, Nucl.Phys. B745 (2006) 84-108, [hep-ph/0602234].

[19] Crystal Ball Collaboration Collaboration, H. Marsiske et al., A Measurement of $\pi^{0} \pi^{0}$ Production in Two Photon Collisions, Phys.Rev. D41 (1990) 3324.

[20] Belle Collaboration Collaboration, S. Uehara et al., High-statistics measurement of neutral pion-pair production in two-photon collisions, Phys.Rev. D78 (2008) 052004, [arXiv: 0805.3387 ].

[21] M. Achasov, K. Beloborodov, A. Berdyugin, A. Bogdanchikov, A. Bozhenok, et al., Experimental study of $\rho \rightarrow \pi^{0} \pi^{0} \gamma$ and $\omega \rightarrow \pi^{0} \pi^{0} \gamma$ decays, Phys.Lett. B537 (2002) 201-210, [hep-ex/0205068].

[22] CMD2 Collaboration, R. Akhmetshin et al., Study of the process $e^{+} e^{-} \rightarrow \pi^{0} \pi^{0} \gamma$ in c.m. energy range 600-MeV to 970-MeV at CMD2, Phys.Lett. B580 (2004) 119-128, [hep-ex/ 0310012 ].

[23] C. Bouchiat and L. Michel, La résonance dans la diffusion méson- $\pi$ méson- $\pi$ et le moment magnétique anormal du méson $\mu$, Journal de Physique et le Radium 22 (1961) 121-121.

[24] L. Durand, Pionic Contributions to the Magnetic Moment of the Muon, Phys.Rev. 128 (1962) 441-448.

[25] M. Davier, A. Hoecker, B. Malaescu, and Z. Zhang, Reevaluation of the Hadronic Contributions to the Muon g-2 and to alpha(MZ), Eur.Phys.J. C71 (2011) 1515, [arXiv: 1010 . 4180].

[26] S. Dubinsky, A. Korchin, N. Merenkov, G. Pancheri, and O. Shekhovtsova, Final-state radiation in electron-positron annihilation into a pion pair, Eur.Phys.J. C40 (2005) 41-54, [hep-ph / 0411113 ]. 